Article

\title{
Emerging Mutations in Nsp1 of SARS-CoV-2 and Their Effect on the Structural Stability
}

\author{
Kejie Mou ${ }^{1}$, Farwa Mukhtar ${ }^{2}$, Muhammad Tahir Khan ${ }^{2, *}$, Doaa B. Darwish ${ }^{3,4} \mathbb{C}^{\text {, Shaoliang Peng }}{ }^{5}$, \\ Shabbir Muhammad ${ }^{6}{ }^{\circ}$, Abdullah G. Al-Sehemi ${ }^{7,8}$ and Dong-Qing Wei ${ }^{5,9, *(0)}$
}

1 Department of Neurosurgery, Bishan Hospital of Chongqing, Chongqing 402760, China; kjb@bsrmyy.cn

2 Institute of Molecular Biology and Biotechnology (IMBB), The University of Lahore, KM Defence Road, Lahore 58810, Pakistan; pbt02203006@student.uol.edu.pk

3 Botany Department, Faculty of Science, Mansoura University, Mansoura 35516, Egypt; ddarwish@ut.edu.sa

4 Department of Biology, Faculty of Science, University of Tabuk, Tabuk 71491, Saudi Arabia

5 Peng Cheng Laboratory, Vanke Cloud City Phase I Building 8, Xili Street, Nashan District, Shenzhen 518055, China; slpeng@hnu.edu.cn

6 Department of Physics, College of Science, King Khalid University, Abha 61413, Saudi Arabia; mshabbir@kku.edu.sa

7 Research Center for Advanced Materials Science (RCAMS), King Khalid University, Abha 61413, Saudi Arabia; agsehemi@kku.edu.sa

8 Department of Chemistry, College of Science, King Khalid University, Abha 61413, Saudi Arabia

9 State Key Laboratory of Microbial Metabolism, Shanghai-Islamabad-Belgrade Joint Innovation Center on Antibacterial Resistances, Joint International Research Laboratory of Metabolic \& Developmental Sciences and School of Life Sciences and Biotechnology, Shanghai Jiao Tong University, Shanghai 200030, China

check for updates

Citation: Mou, K.; Mukhtar, F.; Khan, M.T.; Darwish, D.B.; Peng, S.;

Muhammad, S.; Al-Sehemi, A.G.; Wei, D.-Q. Emerging Mutations in Nsp1 of SARS-CoV-2 and Their Effect on the Structural Stability. Pathogens 2021, 10, 1285. https://doi.org/10.3390/ pathogens10101285

Academic Editors: Roberto Paganelli and Xuguang Li

Received: 27 July 2021

Accepted: 1 October 2021

Published: 6 October 2021

Publisher's Note: MDPI stays neutral with regard to jurisdictional claims in published maps and institutional affiliations.

Copyright: (c) 2021 by the authors. Licensee MDPI, Basel, Switzerland. This article is an open access article distributed under the terms and conditions of the Creative Commons Attribution (CC BY) license (https:/ / creativecommons.org/licenses/by/ $4.0 /)$.
* Correspondence: muhammad.tahir8@imbb.uol.edu.pk (M.T.K.); dqwei@sjtu.edu.cn (D.-Q.W.)

Abstract: The genome of severe acute respiratory syndrome coronavirus 2 (SARS-CoV-2) encodes 16 non-structural (Nsp) and 4 structural proteins. Among the Nsps, Nsp1 inhibits host gene expression and also evades the immune system. This protein has been proposed as a target for vaccine development and also for drug design. Owing to its important role, the current study aimed to identify mutations in Nsp1 and their effect on protein stability and flexibility. This is the first comprehensive study in which 295,000 complete genomes have been screened for mutations after alignment with the Wuhan-Hu-1 reference genome (Accession NC_045512), using the CoVsurver app. The sequences harbored 933 mutations in the entire coding region of Nsp1. The most frequently occurring mutation in the 180-amino-acid Nsp1 protein was R24C $(n=1122)$, followed by D75E $(n=890)$, D48G $(n=881)$, H110Y $(n=860)$, and D144A $(n=648)$. Among the 933 non-synonymous mutations, 529 exhibited a destabilizing effect. Similarly, a gain in flexibility was observed in 542 mutations. The majority of the most frequent mutations were detected in the loop regions. These findings imply that Nsp1 mutations might be useful to exploit SARS-CoV-2's pathogenicity. Genomic sequencing of SARS-CoV-2 on a regular basis will further assist in analyzing variations among the drug targets and to test the diagnostic accuracy. This wide range of mutations and their effect on Nsp1's stability may have some consequences for the host's innate immune response to SARS-CoV-2 infection and also for the vaccines' efficacy. Based on this mutational information, geographically strain-specific drugs, vaccines, and antibody combinations could be a useful strategy against SARS-CoV-2 infection.

Keywords: SARS-CoV-2; genome; mutations; Nsp1

\section{Background}

Severe acute respiratory syndrome 2 (SARS-CoV-2) is responsible for one of the most serious pandemics in human history and has caused huge human and financial losses. There is an urgent need for a molecular framework to determine how the virus deploys the host cellular machinery. Continuous molecular characterization of virus genomes 
from different geographic regions should be investigated to analyze the factors involved, particularly the virus' pathogenicity and its evolutionary phases.

The genome of SARS-CoV-2 encodes 16 non-structural proteins (Nsps) and 4 structural proteins [1]. ORF1ab, the largest component of the virus genome, harboring a high frequency of mutations, splits into $16 \mathrm{Nsps}$ [2,3]. Among these, Nsp1 anchors the replication complex to the cellular membranes as a membrane-associated host translation inhibitor. It also inhibits host translation by forming a complex with a $40 \mathrm{~S}$ ribosomal subunit. Nsp1 degrades the host mRNAs by prompting endonucleolytic cleavage near the $5^{\prime}$-untranslated region [4]. Due to the presence of the $5^{\prime}$-end leader sequence, SARS-CoV-2 mRNAs are not prone to Nsp1-mediated endonucleolytic degradation. The leader protein advances the proficient articulation of viral genes by suppressing the host gene expression in infected cells and inhibiting the host immune response [5].

The Nsp1 of SARS-CoV-2 contains a globular domain (amino acids 13 to 121) between disordered residue locations from 1 to 12 and 122 to 179 [6]. The crystal structure of Nsp1 (PDB ID: $7 \mathrm{k} 7 \mathrm{p}$ ) consists of amino acid (aa) residues from 10 to 126 . The regular Nsp1 secondary structure consists of $\beta 1$ (aa13-20), followed by $\beta 2$ (aa51-54), $\beta 3$ (aa68-73), $\beta 4$ (aa84-92), $\beta 5$ (aa95-97), - $\beta 6$ (aa103-109), and $\beta 7$ (aa117-123), with 310 helixes ( $\eta 1$ and $\eta 2) . \eta 1$ (aa23-25) and $\eta 2$ (aa61-63) are located at the opening, across one barrel, and $\alpha 1$ (aa34-49) is positioned alongside the barrel in contact with $\beta 4$ [6]. In a recent study, the structure of SARS-CoV-2 Nsp1 (aa13-127) was shown to have a feature arrangement of a six-stranded $(n=6) \beta$-barrel, antiparallel except for strands $\beta 1$ (Q15-V20) and $\beta 2$ (C51-V54) [7]. A unique feature of SARS-CoV-2 Nsp1 is the presence of a large number of flexible loops.

Nsp1 is the N-terminal product of ORF1ab, cleaved by papain-like proteinase (PLpro) from the polyprotein, promoting host mRNA degradation, blocking translation, and interfering with the innate immune response [8-11]. It also binds with ribosomal subunits (40S), inserting its C-terminal region into the entrance position of the channel (ribosomal mRNA), blocking the translation of mRNA [12]. The shutdown of the translational process inhibits the innate immune response [13]. Potential drugs may be designed to target the binding pocket, present on the ribosome. The deletion of the Nsp1-coding region results in preventing the cultured cells from becoming infected [6]. Furthermore, mutations in the coding region of Nsp1 may prevent its release from the nascent polyprotein ORF1a, limiting the viability of the virus. More recently [14], mutations in Nsp1 and their association with clinical phenotypes showed that an in-frame deletion in the $\mathrm{N}$-terminal domain is associated with lower IFN- $\beta$ levels in infected patients. The 500-532 region is the most frequent deletion locus across the genome of SARS-CoV-2. The biological importance of the deletion lies in the reduced type I interferon response, highlighting that a mutation in Nsp1 may also affect the host immune response.

We investigated the mutations' frequency and their possible effects on Nsp1 from experimentally determined whole-genome sequences, submitted to the Global Initiative Sharing All Influenza Data (GISAID). These globally collected genome sequences were compared with the SARS-CoV-2 reference genome. This is the first comprehensive study in which 295,000 complete genomes of SARS-CoV-2 were screened for mutations in all amino acid positions of Nsp1. All detected mutations were subjected to analysis of their stability and flexibility effects on Nsp1's structure using the DynaMut server.

\section{Methodology}

\subsection{Genome Sequence Retrieval}

The SARS-CoV-2 genome sequences were retrieved from GISAID (Dec, 2019 to Dec, 2020) (https://www.gisaid.org/, accessed on 30 December 2020) [15]. GISAID shares SARS-CoV-2 genomic data to facilitate their analysis for the purpose of public health and it published both full results and metadata to meet the requirements of public health scientists. A total of 295,000 whole-genome sequences (WGS) have been reported worldwide since December 2020. These WGS data can be downloaded in FASTA format for further analysis, including mutation screening, phylogenetics, and also emerging evolutionary phases of 
SARS-CoV-2. The GISAID server also provides some basic information regarding sequences for data scientists.

\subsection{Genome Sequence Alignment}

All the WGS sequences were aligned with the reference genome of Wuhan-Hu-1 (Accession NC_045512) using the CoVsurver application (https: / /www.gisaid.org/epifluapplications / covsurver-mutations-app/, accessed on 30 December 2020). The CoVsurver is an app of GISAID that aligns query sequences with a reference genome, and the results are displayed in the form of amino acid mutations at specific locations in all structural and Nsp proteins.

All the detected mutations in the Nsp1 of SARS-CoV-2 were organized in Excel sheets. Statistical analysis was performed using EpiData Analysis V2.2.3.187 [16] to screen the most common mutations. EpiData is widely applied to analyze large datasets. The World Health Organization (WHO) uses EpiData for the biostatistical analysis of epidemiological and public health data, and also for other quantitative analyses.

\subsection{Nsp1 Structural Information}

The sharing of infectious agent genomic data is critical to fight against disease outbreaks. The SARS-CoV-2 structural data were retrieved from the Protein Data Bank (PDB) (PDB ID: 7k7p) [17]. PDB (http:/ /www.rcsb.org/pdb/, accessed on 30 December 2020) is a single structural data archive of biological molecules. All the data are primary, collected from depositors across the globe. The data on the macromolecules also contain the coordinates, structures, and methods of structural determination.

\subsection{Missing Residues and Full-Length Nsp1}

The PDB structure of Nsp1 has some missing residues; therefore, we retrieved the full 3D structure of Nsp1 from I-TASSER (ID: QHD43415_1) [18,19]. The chain ID is missing in the I-TASSER 3D structures of Nsp1; therefore, PyMOL (Version 2.3.0 Schrödinger, LLC) Molecular Graphics System was used to add the chain ID for further analysis. ITASSER is most commonly used server, accurately predicting and modeling the protein 3D structure from sequences. The server also provides supporting information, including ligand sites, model validations, and many important residual modeling graphs. The server has already modelled the full-length proteins of SARS-CoV-2 using NCBI reference data (NC_045512) (GenBank MN908947). I-TASSER contains a full-length SARS-CoV-2 protein that is freely available to the academic community (http:/ / zhang.bioinformatics.ku.edu/ I-TASSER, accessed on 30 December 2020). The full-length structure of Nsp1 (ITASSER ID: QHD43415_1) was uploaded to the DynaMut server along with the mutations at each amino acid location one by one. This was done in order to clearly identify the individual effects on the thermodynamic properties of Nsp1.

\subsection{Mutations' Effects on Nsp1 Stability and Flexibility}

Mutations' effects on Nsp1's structural stability and flexibility were computed using the online DynaMut server [20]. Using the server, we implemented the normal-mode methods for the prediction of mutation effects that could be used to analyze the mutations effects on protein stability and flexibility following vibrational entropy changes. The impact of a mutation is predicted through normal-mode dynamics and graph-based signatures. This approach outperforms others $(p$-value $<0.001)$ and the results are also displayed in a good graphical resolution.

\section{Results}

Among the total whole-genome sequences, a large number of mutations were detected in Nsp1, present in the genomes of different geographic regions (Supplementary File S1). All of these mutations were detected using the CoVsurver application (https:/ /www.gisaid. org/epiflu-applications/covsurver-mutations-app/, accessed on 30 December 2020). The 
server is user-friendly and displays the detected mutations in the front column of each protein. The highest frequency of mutations was detected in USA genomic isolates $(n-=233)$, followed by England $(n=201)$, Australia $(n=50)$, and India $(n=27)$. However, the level of transmission and pathogenicity is largely unknown in relation to these mutations. A total of 933 non-synonymous and one synonymous mutation were detected in all 180-amino-acid residue positions of Nsp1 (Supplementary File S1), whereas the frequency of each mutation was different at each of the individual positions. The highest substitution was observed at positions $84(n=10)$ and $85(n=10)$, followed by amino acid positions $17(n=9), 22(n=9)$, and $50(n=9)$. The most common mutation was R24C $(n=1122)$, followed by D75E $(n=890)$, D48G $(n=881)$, H110Y $(n=860)$, and D144A $(n=648)$ (Table 1$)$. These mutations were detected in the flexible loop of the Nsp1 protein. The frequency of the other mutations was also significant (Supplementary File S1). However, the frequency of the N-terminal domain was higher than that of the C-terminal domain of Nsp1.

Table 1. Most common mutations in the Nsp1 of SARS-CoV-2.

\begin{tabular}{|c|c|c|c|c|c|}
\hline Mutation & Frequency & $\begin{array}{c}{ }^{*} \Delta \Delta \mathrm{G}: \\
\mathrm{kcal} / \mathrm{mol}\end{array}$ & Stability & $\begin{array}{c}{ }^{*} \Delta \Delta \text { SVib ENCoM } \\
\text { kcal.mol }^{-1} \cdot \mathrm{K}^{-1}\end{array}$ & Flexibility \\
\hline $\mathrm{R} 24 \mathrm{C}$ & 1122 & -0.218 & Destabilizing & 0.158 & Increase \\
\hline E37D & 237 & -0.844 & Destabilizing & 0.348 & Increase \\
\hline E37K & 309 & -0.243 & Destabilizing & 0.360 & Increase \\
\hline $\mathrm{H} 45 \mathrm{Y}$ & 143 & 1.262 & Stabilizing & -0.217 & Decrease \\
\hline D48G & 881 & -0.217 & Destabilizing & 0.024 & Increase \\
\hline V56I & 113 & 0.954 & Stabilizing & -0.228 & Decrease \\
\hline E57G & 212 & 0.332 & Stabilizing & -0.112 & Decrease \\
\hline V60I & 188 & 0.617 & Stabilizing & -0.192 & Decrease \\
\hline D75E & 890 & 0.31 & Stabilizing & -0.035 & Decrease \\
\hline D75G & 115 & 0.676 & Stabilizing & -0.066 & Decrease \\
\hline M85V & 184 & -0.105 & Destabilizing & 0.687 & Increase \\
\hline E87D & 407 & -1.088 & Destabilizing & 0.395 & Increase \\
\hline L92F & 199 & 0.143 & Stabilizing & -0.002 & Decrease \\
\hline H110Y & 860 & 0.452 & Stabilizing & -0.060 & Decrease \\
\hline $\mathrm{K} 120 \mathrm{~N}$ & 418 & -0.320 & Destabilizing & 0.129 & Increase \\
\hline $\mathrm{R} 124 \mathrm{C}$ & 126 & -1.116 & Destabilizing & 1.056 & Increase \\
\hline N126S & 209 & -0.692 & Destabilizing & 0.177 & Increase \\
\hline D144A & 648 & -1.411 & Destabilizing & 0.415 & Increase \\
\hline S166G & 158 & 1.416 & Stabilizing & 0.225 & Increase \\
\hline
\end{tabular}

\section{Effect of Mutations on Nsp1's Stability and Flexibility}

Mutations often affect the protein's structural properties, including its stability and fluctuations. All of the detected mutations were submitted to the DynaMut server for stability and flexibility analysis. Among the 933 non-synonymous mutations, 529 exhibited a destabilizing effect. Similarly, a gain in flexibility was detected in 542 (Table 2, Supplementary File S1). Amino acid types were considerably different in mutant (MT) Nsp1 when compared with the wild type (WT) (Figure 1). This difference might be useful in Nsp1's function and stability, affecting SARS-CoV-2's pathogenicity and virulency. The most common mutation (R24C), present at the N-terminal end, exhibited a destabilizing effect. Among the most common mutations, eleven exhibited increased flexibility when compared with WT Nsp1 (Table 1), in which the majority were present at the C-terminal domain. The frequencies of these mutations varied from 126 to 1122 . Similarly, a cluster of mutations, present at position 56 to 75 , demonstrated decreased fluctuation. 
Table 2. Frequency of the stability and flexibility effects, predicted for all Nsp1 MTs.

\begin{tabular}{cc}
\hline Stability & Frequency \\
\hline Destabilizing & 529 \\
\hline Stabilizing & 404 \\
\hline Total & 933 \\
\hline Flexibility & 391 \\
\hline Decrease & 542 \\
\hline Increase & 933 \\
\hline Total
\end{tabular}

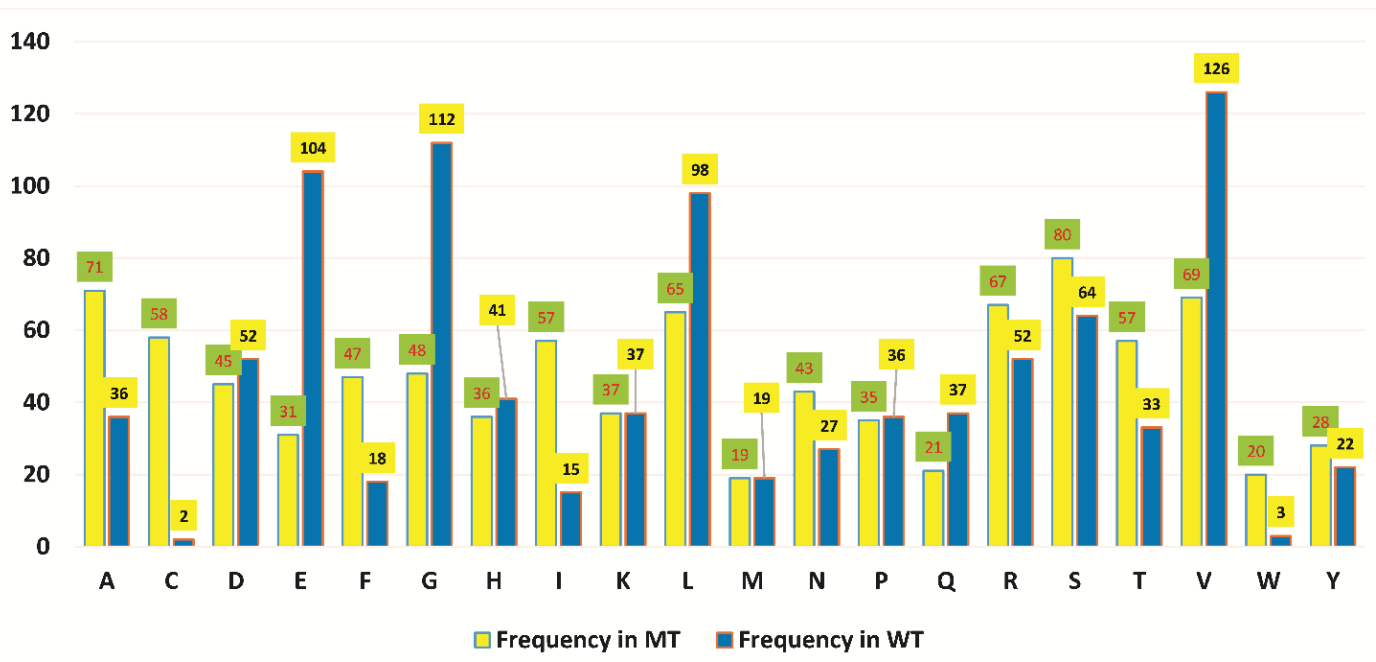

Figure 1. Frequency of amino acids in wild-type (WT) and mutant (MT) Nsp1. Amino acids E, G, L, and V are significantly different in WT and MT. Blue represents WT and yellow represents MT.

The globular domain in Nsp1 (Figure 2), in terms of gaining or losing flexibility in a particular residue, is shown in Figure 3. For the R24C mutant Nsp1, flexibility was altered in the region containing aa residues E65, L64, Q63, P62, C24, D16, and V14. Similarly, in MT D48G Nsp1, a gain in molecular flexibility was detected at aa residues S40, R43, Q44, K47, and G48. A decrease in molecular flexibility was detected in MTs D75E Nsp1 and H110Y (Figure 3), where the blue regions indicate a rigidification and red represents a gain in flexibility. These changes in fluctuation upon non-synonymous mutations might have effects on its normal function in terms of blocking host translation and evading the immune system.

The collective differences in amino acid types in WT and MTs Nsp1, detected in all genomic sequences, are shown (Figure 1). Given the critical role that Nsp1 plays in the SARS-CoV-2 life cycle, amino acid differences in WT and MT may alter the virus' virulency, pathogenicity, and infectivity. The majority of the most frequently occurring mutations were detected in the loop region (Figure 2). Among these, the highest difference was observed in glutamic acid, glycine, isoleucine, and valine.

The most unstable region behind a mutation was detected at aa121 to 124 (Table 3). Numerous mutations at these positions, with various frequencies, were detected. Most of these MTs seem to exert a destabilizing effect, except V121I, V121W, and L122I, present at very low frequencies. 


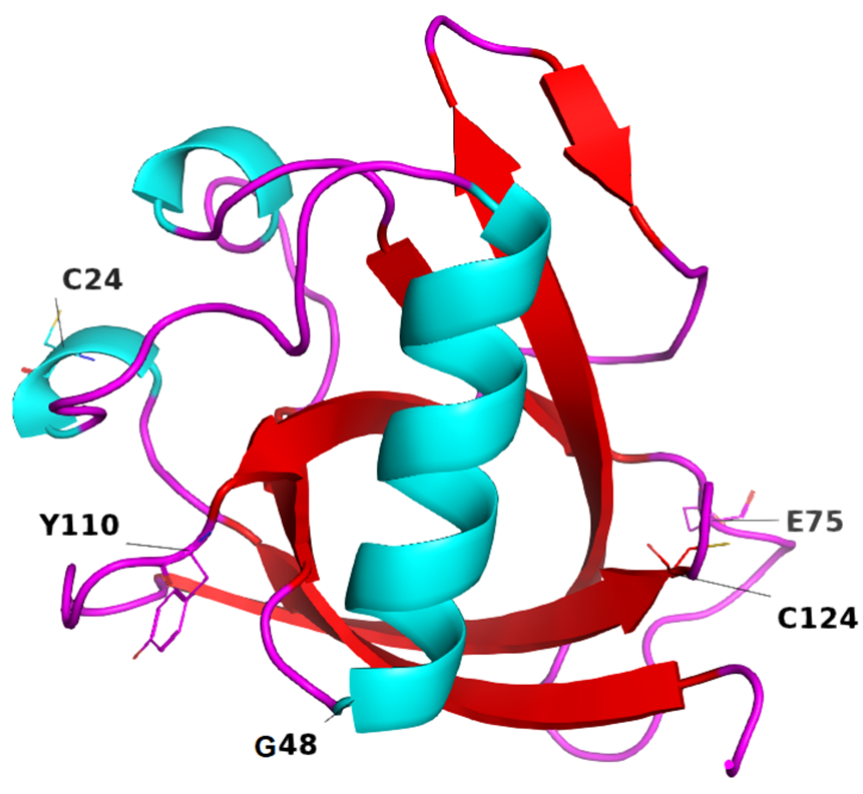

Figure 2. Most frequent mutations in Nsp1 of SARS-CoV-2. Frequency of R24C ( $n=1122), \mathrm{G} 48$ $(n=881)$, D48G $(n=881)$, H110Y $(n=860)$, and R124C $(n=126)$ is shown, present in the loop regions.
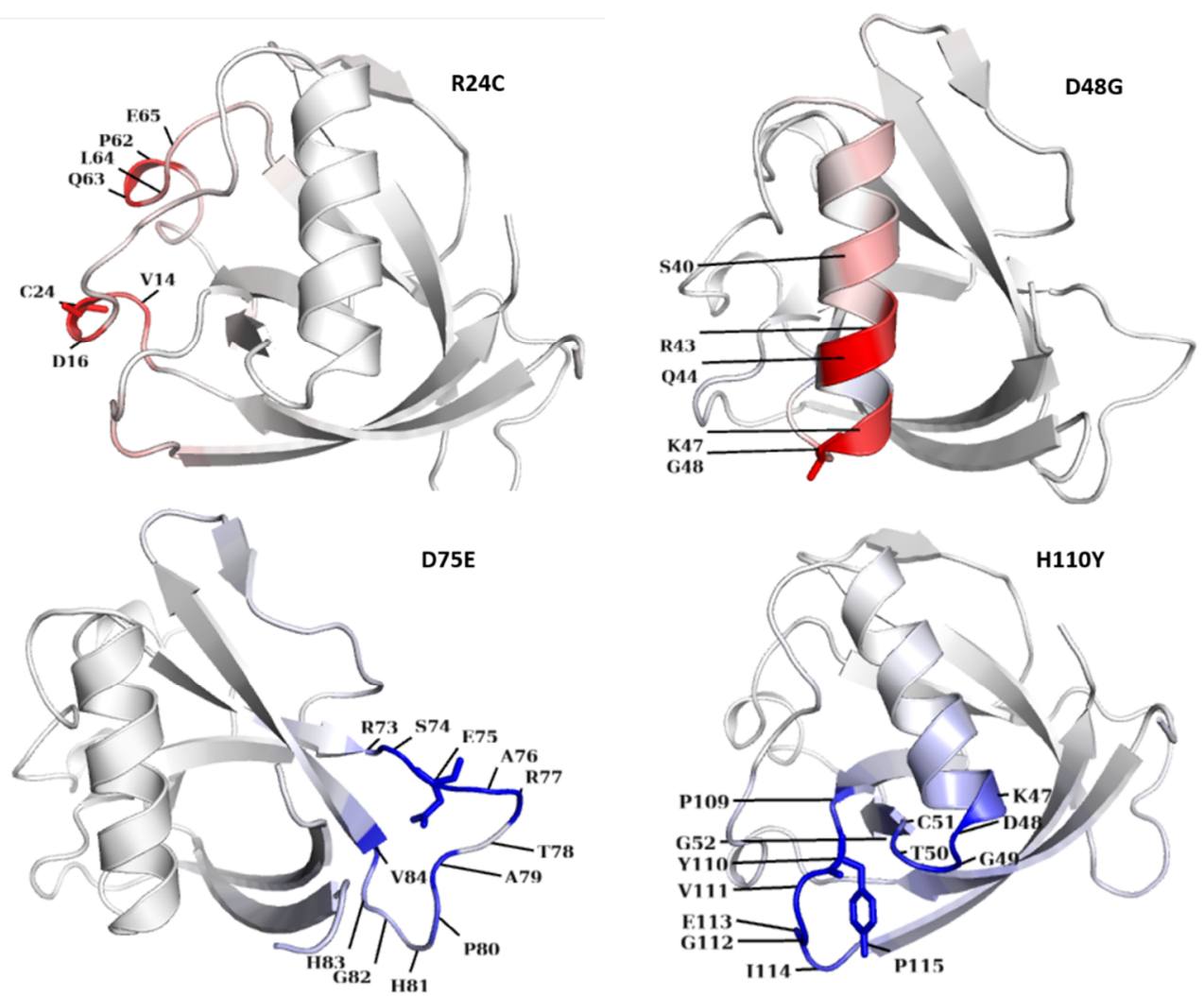

Figure 3. Most common mutations in the Nsp1 globular domain and their effect on flexibility. Residues at red region of Nsp1 show gain in flexibility in common mutations. Blue shows gain in rigidity. Residues at both types of regions are shown. Mutations' sites and their surrounding residues are labelled, showing their flexibility level. 
Table 3. Mutations at positions 121-124 and their effect on Nsp1's stability.

\begin{tabular}{ccccc}
\hline S. NO & Mutation & Frequency & ${ }^{*} \Delta \mathbf{\Delta G} \mathbf{k c a l} / \mathbf{m o l}$ & Stability \\
\hline 1. & V121F & 14 & -0.463 & Destabilizing \\
2. & V121A & 1 & -2.194 & Destabilizing \\
3. & V121D & 1 & -1.469 & Destabilizing \\
4. & V121I & 13 & 0.375 & Stabilizing \\
5. & V121P & 1 & -1.847 & Destabilizing \\
6. & V121W & 1 & 1.136 & Stabilizing \\
7. & L122I & 3 & 0.184 & Stabilizing \\
8. & L122V & 2 & -2.541 & Destabilizing \\
9. & L122F & 21 & -0.577 & Destabilizing \\
10. & L122P & 1 & -1.106 & Destabilizing \\
11. & L122R & 1 & -0.788 & Destabilizing \\
12. & L123F & 14 & -0.784 & Destabilizing \\
13. & L123W & 1 & -1.189 & Destabilizing \\
14. & L123E & 1 & -2.835 & Destabilizing \\
15. & L123I & 1 & -0.175 & Destabilizing \\
16. & R124C & 126 & -1.116 & Destabilizing \\
17. & R124H & 3 & -0.34 & Destabilizing \\
18. & R124V & 2 & -0.096 & Destabilizing \\
\hline
\end{tabular}

* Free energy difference.

\section{Discussion}

Nsp1 has the capacity to redirect proteins that are vital in host translational mechanisms. It is known as the leader protein, also referred to as the "host shutoff factor" and "cellular saboteur" [13,21-26]. Although its role is important in SARS-CoV-2's pathogenesis and evolution, the effect of mutations on Nsp1 activity needs to be investigated for better management of the SARS-CoV-2 infection. Nsp1 likewise prevents the host from collecting an antiviral arsenal [27]. Mutations may affect the virus' pathogenicity, infectivity, and transmission [28-31]. Studies have reported that Nsp1 interferes with the host immune response [8-11] and its deletion results in preventing cultured cells from becoming infected [6]. Moreover, mutations in Nsp1 prevent its release from the nascent polyprotein ORF1a, limiting the viability of the virus. The Nsps are important for vaccine development and as potential drug targets [32,33] because of their role in viral genome replication, disrupting the host cell environment, and interfering with the immune system and signaling [34,35]. SARS-CoV-2 is evolving rapidly and genetic diversity seems to accumulate in a short duration. As per estimations, the substitution mutation rate was high initially [36] and there is a possibility that mutations accumulated during the pandemic could produce measurable effects on the infected population or complicate epidemic control efforts, highlighting a need to monitor the genetic diversity and epidemiology of SARS-CoV-2 throughout the pandemic.

Among all the Nsp1 mutations, 232 and 201 different types were detected in genomic isolates from England and USA, respectively, 50 from Australia and 27 were detected in Indian isolates (Supplementary File S1). According to global statistics, the total number of cases reported till 7 August 2021 in the USA, the UK, Australia, and India was 39.5 million (M), $6.83 \mathrm{M}, 0.56 \mathrm{M}$, and $32.9 \mathrm{M}$, respectively. However, the frequency of Nsp1 mutations from each of these countries was different due the different amounts of wholegenome-sequenced data available in GISAID. The highest frequency of mutations for R24C $(n=1122)$ and D48G $(n=881)$ was detected in SARS-CoV-2 genomic isolates from England; on the contrary, D75E (890) was detected in New Zealand isolates.

Nsp1 obstructs the ribosomal entry site to avert host mRNA binding because it acts as a translation inhibitor via its C-terminal [21]. A SARS-CoV-2 genomic variation has been distinguished with a 9-base-pair deletion in position 686-694, relating to amino acids KSF at position 241-243. This adjusts Nsp1, with a resulting inference in SARS-CoV-2's pathogenesis [37]. Recently, a genome from Bangladesh was found to have some novel mutations, including V121D, which destabilizes Nsp1, inactivating the antiviral system 
mediated by type 1 interferons. Therefore, this MT Nsp1 may be used in attenuated vaccines. Mutations often affect the thermodynamic properties of proteins [38-41]. In the current study, we also detected numerous non-synonymous mutations (V121F $(n=14)$, $\operatorname{V121A}(n=1), \operatorname{V121D}(n=1), \operatorname{V121I}(n=13), \operatorname{V121P}(n=1)$, and V121W $(n=1))$. All these mutations exhibited a destabilizing effect, except for V121I and V121W (Table 3). However, the immune response should be investigated before its subjection to vaccine development. We detected 933 non-synonymous mutations for the first time, among which the majority exhibited a destabilizing effect and increased flexibility. These stability effects behind mutations in Nsp1 might be useful if they weaken the virus' replication competency. In other words, the MT Nsp1 may have a very weak immune response, resulting in a severe infection and pathogenicity. However, mutations' effects must be experimentally verified before their application as attenuated vaccines.

As reported in a recent study, the Nsp1 portion of SARS-CoV-1 and SARS-CoV-2 is well conserved (84\% identity). This high conservation (aa10-126) makes up the crystal structure of Nsp1 (86\%) [6]. However, in the current study, we observed that all of the crystal structure residues harbor numerous mutations in each position (Supplementary File S1). Forty-two different non-synonymous mutations have been detected in the loop (aa9-16). It is well known from previous studies that the loop region of the protein plays a critical role in many interactions. Therefore, mutations in these locations may have a particular effect on their structure and thermodynamic properties [42-46].

Similarly, mutations in loop 2 and $3_{10}$ helixes (aa20-35) were also detected (Supplementary File S1), exhibiting a destabilization effect in the majority of the mutation positions. The most commonly observed mutation was detected at position R24C, present in $3_{10}$ helices (aa20-35). It demonstrated a destabilizing effect and a gain in Nsp1 fluctuations. As previously reported, the $\eta 1$ ( $3_{10}$ helix) is present only in the SARS-CoV-2 structure (aa23-25) and there are no apparent variants in the SARS-CoV-2 $3_{10}$ helix that could stabilize them. However, here, we report that this is a hotspot region for the most frequently occurring mutations (R24C, R24H, R24S, and R24E). Many mutations (R24C, R24H, R24S, R24E, $\mathrm{R} 24 \mathrm{~V}$, and R24A) are also present in the 310 helix, among which $\mathrm{R} 24 \mathrm{C}$ is present with the highest frequency $(n=1122)$ in all Nsp1 mutations, with a destabilizing effect. However, R24S and R24A MTs Nsp1 exhibited a stabilizing effect and a gain in flexibility. All of the $3_{10}$ helix mutations demonstrated an increase in flexibility, except for R24H MT. The effects on flexibility and stability need to be investigated through further experimental approaches for better management of drug design against Nsp1. Point mutations may result in structural perturbations, which may have a pronounced effect on protein flexibility over long distances $[47,48]$.

Recently, four novel mutations have been detected in the genome of SARS-CoV2, among which V121D substitution exhibited a destabilizing effect on Nsp1 [49]. Similar to this study, we detected 18 non-synonymous mutations at positions aa 121 to 124, where all variants exhibited a destabilizing effect, except for V121I, V121W, and L122I. A suitable MT Nsp1 of SARS-CoV-2, which has a major effect on the virus' replication competency, may be promising for use as an attenuated vaccine. The destabilizing effect of MT Nsp1 may be investigated to determine whether the mutations weaken the virus' replication competency or the immune response. In the former case, the MTs may be used as vaccines, while in the latter case, the virus' pathogenicity might be increased. A number of mutations present in the loop and 310 helices exhibited a destabilizing effect in Nsp1 (Supplementary File S1) and this may be investigated for application against SARS-CoV-2 infection. Geography-specific vaccines and drugs may be designed after the careful analysis of the variants in the target proteins for the better management of SARS-CoV-2 infections. According to a recent study, deletion variants in SARS-CoV-2 clinical isolates with related deletions in Nsp1 also induce lower IFN-b responses in infected cells.

Although we screened a large number of genomes for mutations in Nsp1, there are some limitations in the current study. Mutations have not been screened in other Nsps, which might be useful to analyze their association with the virulency of SARS-CoV-2. 
Secondly, the effects of mutations in functionally important sites of Nsps need to be investigated through experimental approaches.

\section{Conclusions}

Mutations are gaining importance due to their role in potential viral pathogenicity and alteration in the host immune response. In the current study, mutations in nearly all amino acid positions of Nsp1 showed that the virus passes through critical stages. This protein exhibited various degrees of stability and flexibility behind mutations in the important loop regions, which might have some consequences for the virus' replication competency and the host immune response. Mutations at amino acid positions 121 to 124 exhibited a high frequency of destabilization, with the exception of V121I, V121W, and L122I, present in very low frequencies.

Further experimental investigations are needed to explore the effect of Nsp1 mutations on virus replication and the immune response that might be useful in diagnosis, and also in vaccine development. More longitudinal studies will further unveil the relationships between Nsp1 mutations and virus replication competency. The host immune response may also be analyzed in infected patients with these MTs. The current study provides useful information for accurate diagnosis and drug design.

Supplementary Materials: The following are available online at https://www.mdpi.com/article/ 10.3390/pathogens10101285/s1, Supplementary File S1: List of mutations and their effect on Nsp1 stability and flexibility.

Author Contributions: Conception and design of the study: M.T.K.; Acquisition of data: F.M., D.B.D.; Analysis and interpretation of data: S.M., S.P.; Drafting the article: K.M., M.T.K.; Revising content: D.-Q.W., A.G.A.-S.; Final approval of the version: D.-Q.W., M.T.K.; Funding: K.M. All authors have read and agreed to the published version of the manuscript.

Funding: Dong-Qing Wei was supported by grants from the National Science Foundation of China (Grant No. 32070662, 61832019, 32030063), the Key Research Area Grant 2016YFA0501703 of the Ministry of Science and Technology of China, and the Science and Technology Commission of Shanghai Municipality (Grant No. 19430750600). Kejie Mou was supported by the Key project of science and technology research of Chongqing Municipal Education Commission (kjzd-k201902802), the Project of the Chongqing Education Science Research Program for the 13th Five-year Plan (2018-GX-462), the Key project of education and teaching reform in Chongqing Medical and Pharmaceutical College (CQYGZJG1909), and the Talent introduction project of Chongqing Medical and Pharmaceutical College (ygz2016302). The authors from King Khalid University extend their appreciation to the Deanship of Scientific Research at King Khalid University for funding the work through the research project RGP.2/128/42.

Institutional Review Board Statement: Not applicable.

Informed Consent Statement: Not applicable.

Data Availability Statement: The datasets in the current study are available as Supplementary File S1 and can be freely accessed from GISAID (https://www.gisaid.org/).

Acknowledgments: The authors are thankful to Aamer Iqbal Bhatti and the University of Lahore's management section for their technical support.

Conflicts of Interest: The authors declare no conflict of interest.

\section{Abbreviations}

GISAID Global Initiative Sharing All Influenza Data

Nsp Non-structural protein

ORF Open reading frame

SARS-CoV Severe acute respiratory syndrome coronavirus

SARS-CoV-2 Severe acute respiratory syndrome coronavirus 2 
(COVID-19) Coronavirus disease 19

MT Mutants

PDB Protein Data Bank

WT Wild type

\section{References}

1. Guo, Y.-R.; Cao, Q.-D.; Hong, Z.-S.; Tan, Y.-Y.; Chen, S.-D.; Jin, H.-J.; Tan, K.-S.; Wang, D.-Y.; Yan, Y. The Origin, Transmission and Clinical Therapies on Coronavirus Disease 2019 (COVID-19) Outbreak-an Update on the Status. Mil. Med. Res. 2020, 7, 1-10. [CrossRef]

2. Phan, T. Genetic Diversity and Evolution of SARS-CoV-2. Infect. Genet. Evol. 2020, 81, 104260. [CrossRef]

3. Khailany, R.A.; Safdar, M.; Ozaslan, M. Genomic Characterization of a Novel SARS-CoV-2. Gene Rep. 2020, 19, 100682. [CrossRef] [PubMed]

4. Lokugamage, K.G.; Narayanan, K.; Huang, C.; Makino, S. Severe Acute Respiratory Syndrome Coronavirus Protein Nsp1 Is a Novel Eukaryotic Translation Inhibitor That Represses Multiple Steps of Translation Initiation. J. Virol. 2012, 86, 13598-13608. [CrossRef]

5. Gottipati, K. Membrane Binding and Rearrangement by Chikungunya Virus Capping Enzyme NsP1. Virology 2020, 544, 31-41. [CrossRef] [PubMed]

6. Clark, L.K.; Green, T.J.; Petit, C.M. Structure of Nonstructural Protein 1 from SARS-CoV-2. J. Virol. 2021, 95. [CrossRef] [PubMed]

7. Semper, C.; Watanabe, N.; Savchenko, A. Structural Characterization of Nonstructural Protein 1 from SARS-CoV-2. iScience 2021, 24, 101903. [CrossRef] [PubMed]

8. Chaudhuri, A. Comparative Analysis of Non Structural Protein 1 of SARS-COV2 with SARS-COV1 and MERS-COV: An in Silico Study. bioRxiv 2020, bioRxiv:2020.06.09.142570. [CrossRef]

9. Huang, C.; Lokugamage, K.G.; Rozovics, J.M.; Narayanan, K.; Semler, B.L.; Makino, S. SARS Coronavirus Nsp1 Protein Induces Template-Dependent Endonucleolytic Cleavage of MRNAs: Viral MRNAs Are Resistant to Nsp1-Induced RNA Cleavage. PLoS Pathog. 2011, 7, e1002433. [CrossRef]

10. Narayanan, K.; Ramirez, S.I.; Lokugamage, K.G.; Makino, S. Coronavirus Nonstructural Protein 1: Common and Distinct Functions in the Regulation of Host and Viral Gene Expression. Virus Res. 2015, 202, 89-100. [CrossRef] [PubMed]

11. Shen, Z.; Wang, G.; Yang, Y.; Shi, J.; Fang, L.; Li, F.; Xiao, S.; Fu, Z.F.; Peng, G. A Conserved Region of Nonstructural Protein 1 from Alphacoronaviruses Inhibits Host Gene Expression and Is Critical for Viral Virulence. J. Biol. Chem. 2019, 294, 13606-13618. [CrossRef] [PubMed]

12. Min, Y.-Q.; Huang, M.; Sun, X.; Deng, F.; Wang, H.; Ning, Y.-J. Immune Evasion of SARS-CoV-2 from Interferon Antiviral System. Comput. Struct. Biotechnol. J. 2021, 19, 4217-4225. [CrossRef] [PubMed]

13. Thoms, M.; Buschauer, R.; Ameismeier, M.; Koepke, L.; Denk, T.; Hirschenberger, M.; Kratzat, H.; Hayn, M.; Mackens-Kiani, T.; Cheng, J. Structural Basis for Translational Shutdown and Immune Evasion by the Nsp1 Protein of SARS-CoV-2. Science 2020, 369, 1249-1255. [CrossRef] [PubMed]

14. Lin, J.; Tang, C.; Wei, H.; Du, B.; Chen, C.; Wang, M.; Zhou, Y.; Yu, M.; Cheng, L.; Kuivanen, S.; et al. Genomic Monitoring of SARS-CoV-2 Uncovers an Nsp1 Deletion Variant That Modulates Type I Interferon Response. Cell Host Microbe 2021, 29 , 489-502. [CrossRef] [PubMed]

15. Elbe, S.; Buckland-Merrett, G. Data, Disease and Diplomacy: GISAID's Innovative Contribution to Global Health. Glob. Chall. 2017, 1, 33-46. [CrossRef] [PubMed]

16. EpiData Software-Http://Www.Epidata.Dk. Available online: http://www.epidata.dk/download.php (accessed on 17 November 2017).

17. Berman, H.M.; Westbrook, J.; Feng, Z.; Gilliland, G.; Bhat, T.N.; Weissig, H.; Shindyalov, I.N.; Bourne, P.E. The Protein Data Bank. Nucleic Acids Res. 2000, 28, 235-242. [CrossRef] [PubMed]

18. Roy, A.; Kucukural, A.; Zhang, Y. I-TASSER: A Unified Platform for Automated Protein Structure and Function Prediction. Nat. Protoc. 2010, 5, 725-738. [CrossRef]

19. Zhang, Y. I-TASSER Server for Protein 3D Structure Prediction. BMC Bioinform. 2008, 9, 40. [CrossRef] [PubMed]

20. Rodrigues, C.H.; Pires, D.E.; Ascher, D.B. DynaMut: Predicting the Impact of Mutations on Protein Conformation, Flexibility and Stability. Nucleic Acids Res. 2018, 46, W350-W355. [CrossRef] [PubMed]

21. Schubert, K.; Karousis, E.D.; Jomaa, A.; Scaiola, A.; Echeverria, B.; Gurzeler, L.-A.; Leibundgut, M.; Thiel, V.; Mühlemann, O.; Ban, N. SARS-CoV-2 Nsp1 Binds the Ribosomal MRNA Channel to Inhibit Translation. Nat. Struct. Mol. Biol. 2020, 27, 959-966. [CrossRef] [PubMed]

22. Shi, M.; Wang, L.; Fontana, P.; Vora, S.; Zhang, Y.; Fu, T.-M.; Lieberman, J.; Wu, H. SARS-CoV-2 Nsp1 Suppresses Host but Not Viral Translation through a Bipartite Mechanism. bioRxiv 2020. [CrossRef]

23. Vankadari, N.; Jeyasankar, N.N.; Lopes, W.J. Structure of the SARS-CoV-2 Nsp1/5'-Untranslated Region Complex and Implications for Potential Therapeutic Targets, a Vaccine, and Virulence. J. Phys. Chem. Lett. 2020, 11, 9659-9668. [CrossRef] [PubMed] 
24. Narayanan, K.; Huang, C.; Lokugamage, K.; Kamitani, W.; Ikegami, T.; Tseng, C.-T.K.; Makino, S. Severe Acute Respiratory Syndrome Coronavirus Nsp1 Suppresses Host Gene Expression, Including That of Type I Interferon, in Infected Cells. J. Virol. 2008, 82, 4471-4479. [CrossRef]

25. Tohya, Y.; Narayanan, K.; Kamitani, W.; Huang, C.; Lokugamage, K.; Makino, S. Suppression of Host Gene Expression by Nsp1 Proteins of Group 2 Bat Coronaviruses. J. Virol. 2009, 83, 5282-5288. [CrossRef] [PubMed]

26. Corum, J.; Zimmer, C. Bad News Wrapped in Protein: Inside the Coronavirus Genome. The New York Times. 3 April 2020. Available online: https:/ / www.nytimes.com/interactive/2020/04/03/science/coronavirus-genome-bad-news-wrapped-in-protein.html (accessed on 20 December 2020).

27. Bai, Y.; Yao, L.; Wei, T.; Tian, F.; Jin, D.-Y.; Chen, L.; Wang, M. Presumed Asymptomatic Carrier Transmission of COVID-19. JAMA 2020, 323, 1406-1407. [CrossRef] [PubMed]

28. Khan, M.T.; Ali, S.; Khan, A.S.; Muhammad, N.; Khalil, F.; Ishfaq, M.; Irfan, M.; Al-Sehemi, A.G.; Muhammad, S.; Malik, A.; et al. SARS-CoV-2 Genome from the Khyber Pakhtunkhwa Province of Pakistan. ACS Omega 2021, 6, 6588-6599. [CrossRef] [PubMed]

29. Islam, O.K.; Al-Emran, H.M.; Hasan, M.S.; Anwar, A.; Jahid, M.I.K.; Hossain, M.A. Emergence of European and North American Mutant Variants of SARS-CoV-2 in South-East Asia. Transbound. Emerg. Dis. 2020, 68, 824-832. [CrossRef] [PubMed]

30. Tegally, H.; Wilkinson, E.; Giovanetti, M.; Iranzadeh, A.; Fonseca, V.; Giandhari, J.; Doolabh, D.; Pillay, S.; San, E.J.; Msomi, N.; et al. Detection of a SARS-CoV-2 Variant of Concern in South Africa. Nature 2021, 592, 438-443. [CrossRef] [PubMed]

31. Daniloski, Z.; Jordan, T.X.; Ilmain, J.K.; Guo, X.; Bhabha, G.; tenOever, B.R.; Sanjana, N.E. The Spike D614G Mutation Increases SARS-CoV-2 Infection of Multiple Human Cell Types. eLife 2021, 10, e65365. [CrossRef]

32. Khan, M.T.; Ali, A.; Wang, Q.; Irfan, M.; Khan, A.; Zeb, M.T.; Zhang, Y.-J.; Chinnasamy, S.; Wei, D.-Q. Marine Natural Compounds as Potents Inhibitors against the Main Protease of SARS-CoV-2. A Molecular Dynamic Study. J. Biomol. Struct. Dyn. 2021, 39, 3627-3637. [CrossRef]

33. Khan, A.; Tahir Khan, M.; Saleem, S.; Junaid, M.; Ali, A.; Shujait Ali, S.; Khan, M.; Wei, D.-Q. Structural Insights into the Mechanism of RNA Recognition by the N-Terminal RNA-Binding Domain of the SARS-CoV-2 Nucleocapsid Phosphoprotein. Comput. Struct. Biotechnol. J. 2020, 18, 2174-2184. [CrossRef] [PubMed]

34. Biswas, N.; Kumar, K.; Mallick, P.; Das, S.; Kamal, I.M.; Bose, S.; Choudhury, A.; Chakrabarti, S. Structural and Drug Screening Analysis of the Non-Structural Proteins of Severe Acute Respiratory Syndrome Coronavirus 2 Virus Extracted From Indian Coronavirus Disease 2019 Patients. Front. Genet. 2021, 12, 171. [CrossRef]

35. Khan, M.T.; Zeb, M.T.; Ahsan, H.; Ahmed, A.; Ali, A.; Akhtar, K.; Malik, S.I.; Cui, Z.; Ali, S.; Khan, A.S.; et al. SARS-CoV-2 Nucleocapsid and Nsp3 Binding: An in Silico Study. Arch. Microbiol. 2021, 203, 59-66. [CrossRef]

36. van Dorp, L.; Richard, D.; Tan, C.C.S.; Shaw, L.P.; Acman, M.; Balloux, F. No Evidence for Increased Transmissibility from Recurrent Mutations in SARS-CoV-2. Nat. Commun. 2020, 11, 5986. [CrossRef]

37. Benedetti, F.; Snyder, G.A.; Giovanetti, M.; Angeletti, S.; Gallo, R.C.; Ciccozzi, M.; Zella, D. Emerging of a SARS-CoV-2 Viral Strain with a Deletion in Nsp1. J. Transl. Med. 2020, 18, 1-6. [CrossRef]

38. Khan, M. Pyrazinamide Resistance and Mutations L19R, R140H, and E144K in Pyrazinamidase of Mycobacterium Tuberculosis. J. Cell. Biochem. P 2019, 120, 7154-7166. [CrossRef] [PubMed]

39. Khan, M.T.; Rehaman, A.U.; Junaid, M.; Malik, S.I.; Wei, D.-Q. Insight into Novel Clinical Mutants of RpsA-S324F, E325K, and G341R of Mycobacterium Tuberculosis Associated with Pyrazinamide Resistance. Comput. Struct. Biotechnol. J. 2018, 16, 379-387. [CrossRef]

40. Malickbasha, M.; Arunachalam, R.; Senthilkumar, B.; Rajasekarapandian, M.; Annadurai, G. Effect of OmpR Gene Mutation in Expression of OmpC and OmpF of Salmonella Typhi. Interdiscip. Sci. Comput. Life Sci. 2010, 2, 157-162. [CrossRef]

41. Nakano, M.; Watanabe, H.; Starikov, E.B.; Rothstein, S.M.; Tanaka, S. Mutation Effects on Structural Stability of Polyglutamine Peptides by Molecular Dynamics Simulation. Interdiscip. Sci. Comput. Life Sci. 2009, 1, 21-29. [CrossRef] [PubMed]

42. Siegert, T.R.; Bird, M.J.; Makwana, K.M.; Kritzer, J.A. Analysis of Loops That Mediate Protein-Protein Interactions and Translation into Submicromolar Inhibitors. J. Am. Chem. Soc. 2016, 138, 12876-12884. [CrossRef] [PubMed]

43. Gavenonis, J.; Sheneman, B.A.; Siegert, T.R.; Eshelman, M.R.; Kritzer, J.A. Comprehensive Analysis of Loops at Protein-Protein Interfaces for Macrocycle Design. Nat. Chem. Biol. 2014, 10, 716-722. [CrossRef] [PubMed]

44. Nemtseva, E.V.; Gerasimova, M.A.; Melnik, T.N.; Melnik, B.S. Experimental Approach to Study the Effect of Mutations on the Protein Folding Pathway. PLoS ONE 2019, 14, e0210361. [CrossRef] [PubMed]

45. Bhattacharya, R.; Rose, P.W.; Burley, S.K.; Prlić, A. Impact of Genetic Variation on Three Dimensional Structure and Function of Proteins. PLOS ONE 2017, 12, e0171355. [CrossRef]

46. Shanthirabalan, S.; Chomilier, J.; Carpentier, M. Structural Effects of Point Mutations in Proteins. Proteins Struct. Funct. Bioinforma. 2018, 86, 853-867. [CrossRef] [PubMed]

47. Verma, D.; Jacobs, D.J.; Livesay, D.R. Changes in Lysozyme Flexibility upon Mutation Are Frequent, Large and Long-Ranged. PLoS Comput. Biol. 2012, 8, e1002409. [CrossRef] [PubMed]

48. Iyer, K.A.; Hu, Y.; Nayak, A.R.; Kurebayashi, N.; Murayama, T.; Samsó, M. Structural Mechanism of Two Gain-of-Function Cardiac and Skeletal RyR Mutations at an Equivalent Site by Cryo-EM. Sci. Adv. 2020, 6, eabb2964. [CrossRef] [PubMed]

49. Hossain, M.U.; Bhattacharjee, A.; Emon, M.T.H.; Chowdhury, Z.M.; Mosaib, M.G.; Moniruzzaman, M.; Rahman, M.H.; Islam, M.N.; Ahmed, I.; Amin, M.R.; et al. Novel Mutations in NSP1 and PLPro of SARS-CoV-2 NIB-1 Genome Mount for Effective Therapeutics. bioRxiv 2020, bioRxiv:2020.12.02.408229. [CrossRef] 